\title{
The Effect of a Cementless Stem Cross-Sectional Shape on Mechanical Stability: A Finite Element Analysis approach
}

\author{
Rahul Ribeiro ${ }^{1}$, Beni Ram Rawal ${ }^{2}$, Naresh Bhatnagar ${ }^{3}$ \\ Associate Professor, Department of Mechanical Engineering, Alliance University, Bengaluru, India ${ }^{1}$ \\ Associate Professor, Department of Mechanical Engg., Shri G.S. Institute of Technology and Science, Indore, India ${ }^{2}$ \\ Professor, Department of Mechanical Engineering, Indian Institute of Technology, Delhi, New Delhi, India ${ }^{3}$
}

\begin{abstract}
The standard cementless femoral stems commercially available may not be the best-fit to a patient in markets like India, because of the large anatomic variation of the human hip joint among the population. This investigation presents a regional anthropometric data based design approach for the standard anatomical cementless femoral component used in total hip replacement (THR). Aseptic loosening and dislocation are the most important causes of failure of THR due to the mismatch in dimensions between the femur bone and stem. Standard cementless stems with different cross-section shapes were designed and analyzed to solve the problem of a possible geometric mismatch between a selected implant and the hip joint considering a variety of patients with differing anatomical sizes. Finite element analysis was conducted on cementless tapered stems with four different stem cross-sectional shapes - oval, trapezoidal, rectangular and wedge. Comparison of Von Mises stresses and micromotion under axial and torsional loads were made. This study indicates a need for a regional anthropometric data based design of cementless femoral stems for providing greater longevity and better recovery to patients. This novel design approach can improve the implant fixation enhancing primary stability, rigidity, longevity of the implant and relieving patients from discomfort.
\end{abstract}

Keywords: Total hip replacement, cementless stem, mechanical stability, cross section.

\section{INTRODUCTION}

In THR, bone cement has traditionally been used. However, in the 1980's a cementless design was developed [1]. The cementless designs are porous or coated implants. The intent is, through biologic fixation, bone grows into the pores in the implant, thereby securing it firmly. In theory, the cementless joint replacements are expected to reduce the chance of infection and loosening of the prosthesis, which are two major complications of hip replacement surgery using bone cement. Recent research, however, indicates that both the cemented and cementless joints perform very well, but the problem of loosening still exists with the cementless implants among young people with a more active lifestyle [2]. It has been also found that the geometry of the proximal femur is determined by genetic and environmental factors such as age, race, gender and lifestyle [3-4]. Several researchers have stressed the need for a proper implant-patient match in hip joint replacements, in particular, for cementless femoral stems [5-7]. This calls for a best-fit cementless hip implant design. Modular stems can be designed, which are patient specific but are very expensive. It is therefore necessary to design a cementless hip joint prosthesis stem that would fit a number of patients, with minimal chance of loosening. In the last few decades, studies have been carried out by finite element analysis, to study the effect of geometric parameters and optimization of cross-section for prostheses design [8-10]. The studies focused on optimizing the shape of a cemented stem with the objective of minimizing the stresses in the cement. This works were part of the efforts that led to a new cemented

stem. However, these efforts have not helped much in designing and optimizing a cementless stem, because the cementless stem designs are larger and longer than those used with cement. Many other studies have also applied shape optimization to cemented hip stem design, [11-13]. Kowalczyk et. al. [14] presented an optimization procedure to minimize the stresses on the bone-stem interface of cementless stems. Ruben et. al.[15] presented structural optimization techniques to obtain cementless stem shapes with improved initial stability and implant performance by minimizing bone-implant interfacial micromotions. Kowalczyk et. al. [14] and Ruben et. al. [15] have described the procedure to optimized the geometries for the femoral component of hip prosthesis to design custom-made hip prostheses that depend on a patient's personal femur geometry.

Stress distribution within the bone and implant and relative motions between the bone and implant depend mainly on loading conditions and on the implant design, (i.e., its mechanical properties and shape). As the latter is easy to modify over a wide range, the design optimization of the implant cross-section appears to be a promising way to improve the longevity and functionality of hip joint prostheses. Therefore there is a need to optimize the cementless hip implant with the appropriate cross- section, which would not only aid in the best bone- implant interface, but also maintain appropriate strength and rigidity, enhance implant fixation, and offer better mobility to the patient. This study deals with the comparison of Von Mises stresses and micromotions 
under axial and torsional loads for different cementless hip stem cross- sections such as oval, trapezoidal, rectangular and wedge. The optimization of the cross -section was performed for the hip implant design which had minimum and uniform stress distribution and minimum micromotion at the bone implant interface.

\section{METHODS}

\section{A. Measurement of anthropometric parameters}

There are certain geometrical parameters of the hip joint which specify the overall shape of the joint and enable designing a best fit standard femoral stem for cementless insertion. Geometric dimensions were extracted from 56 left and 42 right femurs which belonged to 29 female and 31 male subjects (both femurs of a few patients and a single femur of remaining). The age of the total population ranged from 40 to 81 years with an average age of 61.3 years. This sample population included patients from different regions of India. CT- scanned images were obtained in DICOM (Digital Imaging and Communications in Medicine) format from the department of orthopedics, All India Institute of Medical Sciences, New Delhi, India. The patients had reported with complications with hip joint function. Necessary consent was obtained from the patients. The slice thickness of the scans was $1.25 \mathrm{~mm}$.

The geometrical parameters of the femur that were chosen to be extracted for understanding the anthropometry included femoral head offset, femoral head diameter, femoral head position, position of shaft isthmus, periosteal width at the isthmus, neck-shaft angle, femoral neck length, and mediolateral and anteroposterial canal width.

\section{B. Design methodology}

The first stage in the current design was the development of a general geometry that restores, as much as possible, the natural load-transfer mechanism through the proximal femur. The cross-sections were designed to be representative of the anthropometric data of the population.

The range for the particular parameter is based on anthropometric measurements and statistical analysis. The range of these geometric parameters is given in Table 1. The implant was designed so that different cross-sectional geometries could be swept along the guide profile of the implant (Fig. 1a). The first cross-section, designated crosssection A, had an oval cross-section. The second crosssection, labeled $\mathrm{B}$, was a variation on cross-section $\mathrm{A}$, with a medial surface that is half the diameter of that of the lateral surface i.e. wedge (Fig. 1c). Cross-section $\mathrm{C}$ was trapezoidal. Cross-section D was rectangular. All the cross-sections had the same width and length at both ends (as shown in Figs.1b, c, d \& e).

The femoral geometric axis and neck axes were merged with a smooth curve as shown in the model below in Fig. 1a. The curve diameter and centre were chosen so as to provide a proper fit during implantation.

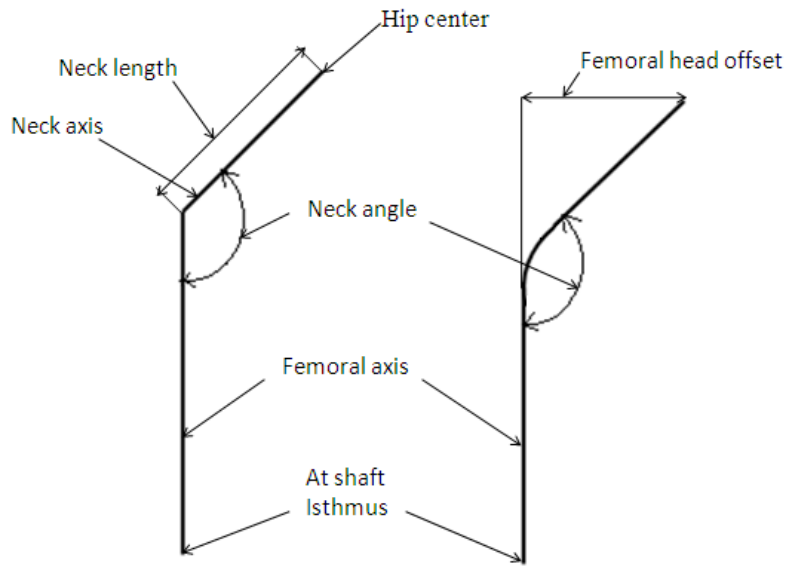

Fig. 1a Stem anatomy and profile guide curve geometry
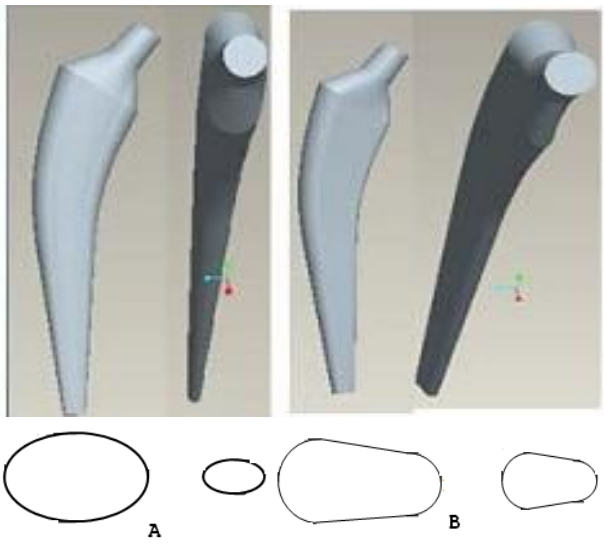

$1 b$

$1 \mathrm{c}$

Fig. $1 \mathrm{~b}$ and c. Oval and wedge cross sections
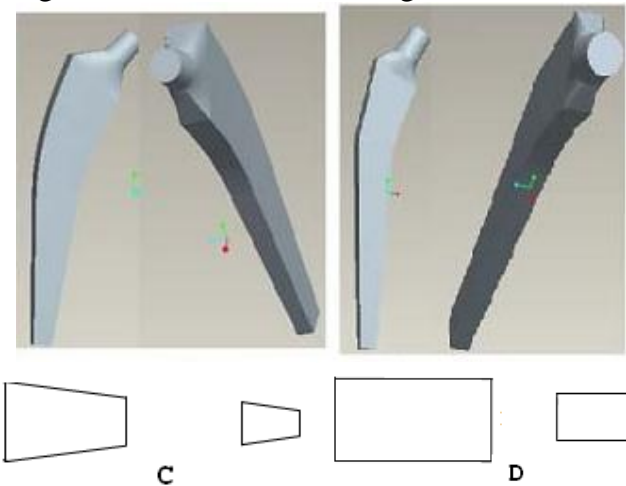

$c$



$1 d$

$1 \mathrm{e}$

Fig. 1d and e. Trapezoidal and wedge cross sections A comparative analysis was performed to determine which geometrical cross section produces the minimum and most uniform von-Mises stresses, and the lowest micromovement, thus having the potential for longer life. The base implant is designed to be an anatomical cementless hip implant. Various models were generated designed with different cross-sectional geometries and dimensions using Pro-e software.

Virtual implantations of the designed stems were performed with all digitized virtual femur 3D models of the CT scans, to adjust an anatomically shaped cementless stem to the proximal femur anatomy by repeated virtual implantations as shown in Fig. 2. In anatomically different 
femurs, the shape of the stem was improved step by step E. Material properties

according to the virtual implantation protocol. A The cortical portion was modeled as a linear, elastic simulation was performed according to the virtual stem tubular structure composed of isotropic bone (modulus of implantation protocol outlined by Adam et. al. [16]. Based elasticity of $17.0 \mathrm{GPa}$, and a Poisson's ratio of 0.3) [8]. on fit analysis protocol and mean total contact area, the Since the trabeculae bone was assumed to be rasped prior best cross-section was chosen. The "Contact" (a mean to implantation, it was assumed to be highly compressed, percent of stem area contact with cortical bone) is defined as a bone-prosthesis distance less than $0.5 \mathrm{~mm}$.
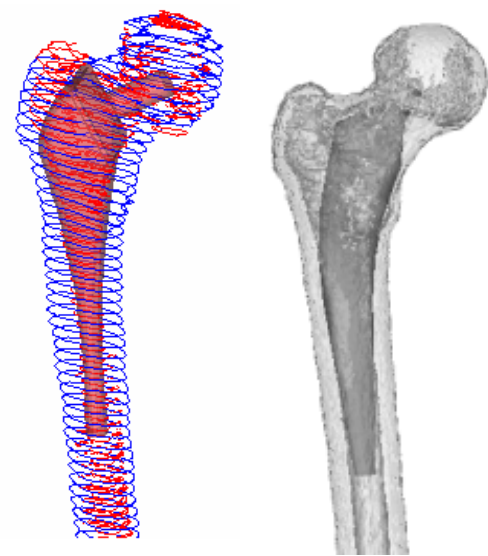

Fig. 2. Virtual implantation of designed femoral stem.

\section{Finite element model}

Finite element analysis software ANSYS Workbench 11 was used for carrying out the analysis. In this study, static structural analysis was carried out using 3D solid tetrahedron elements for 3D analysis. They were fournode tetrahedrons with linear shape functions. Each complete model consisted of around 71,537 elements and 85,600 nodes. The femur consisted of 15,800 elements including both trabecular and cortical bone and the implant consisted of 41,100 elements. The rest of about 14,637 elements were used for contact and target elements. A fine mesh was applied to the implant trabeculae interface.

Convergence tests were applied to capture the accurate value of stresses and sliding motion (micromotions). Fine meshing was done and the element size of the models was reduced from $4 \mathrm{~mm}$ to $1.5 \mathrm{~mm}$ in steps of $0.5 \mathrm{~mm}$ until desired $5 \%$ change in output, convergence criteria was achieved. The femur-implant interface area was meshed with elements having an average size of $0.5 \mathrm{~mm}$. This fine mesh was used to obtain accurate results in the primary area of interest around the femur-implant interface. The rest of the model was meshed with elements having an average size of $2 \mathrm{~mm}$.

D. Interface conditions

Standard surface to surface frictional contact was defined between the implant and bone using contact elements CONTA174 and TARGE170. The coefficient of friction value of 0.34 was used in this study, based on literature reference [17]. The augmented Lagrange method was used for solving the contact problem. Simulations were carried out with normal stiffness factors (FKN) of 0.1 and 0.15 . Furthermore, another problem is the choice of the threshold value for micromotions. In this work, a value of $50 \mu \mathrm{m}$ (microns) was used as a conservative but fair value according to Engh et al. [17]. with the result that its properties became equal to that of cortical bone. Each femoral stem design was made of titanium alloy Ti6Al4V, having a Young's modulus of 117 $\mathrm{GPa}$ and a Poisson's ratio of 0.33 .

F. Loading and boundary conditions

In ANSYS software, the implant was implanted into a femur (processed from CT scans), belonging to a male patient, age 71 years. The dimensions of the rasp tool was $1 \mathrm{~mm}$ less than those of the implant, therefore, a press-fit was assumed for the implantation.

For static stress analysis, the femoral stem designs were analyzed at compressive forces ranging from 2.5 to $7 \mathrm{kN}$ ( $\mathrm{F}$ in Fig. 3a), in steps of $1000 \mathrm{~N}$, applied in the frontal plane at an angle of $20^{\circ}$ (with vertical) in a mediolateral direction on the centre of the neck top surface of the stem, as shown in Fig. 3a. This load is representative of a person weighing $70 \mathrm{~kg}$ [18]. An abductor muscle load of $1.25 \mathrm{kN}$ (MF abductor muscle) is applied at an angle of $20^{\circ}$ to the proximal area of the greater trochanter as shown in Fig.3c. Torsional loads from 0 to $40 \mathrm{Nm}$, in steps of $10 \mathrm{Nm}$ (Transverse force $\mathrm{x}$ femoral head offset) were applied in an anteroposterior direction in the transverse plane again at the tip of stem as shown Fig. 3b. These forces were selected as a typical gait cycle generates forces up to 6-7 times the body weight in the hip joint [19]. The model test assembly was constrained at the bottom in all directions as shown in Fig.3c.

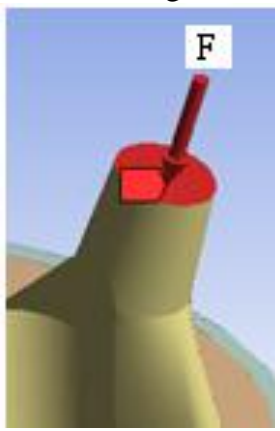

(a) Force in

mediolateral direction

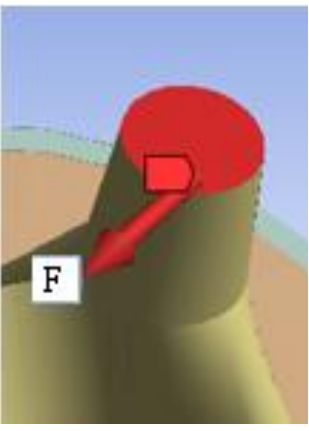

(b) Forces that exert torque in anteroposterial direction

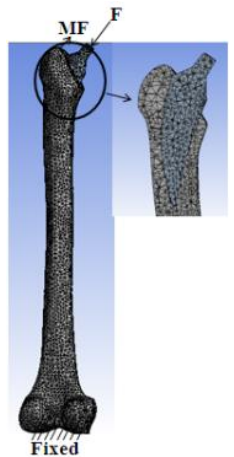

(c) Meshing on test assembly
Fig. 3 Loading and boundary conditions on test assembly

\section{III.RESULTS}

The geometric shape of the cementless femoral stem was devised and actualized based on extracted Indian anthropometric data. The geometrical parameters should be in the design range for a better fitment. The range of these geometric parameters based on anthropometric measurements and statistical analysis of deviations in the given sample size of 98 femurs (both male and female subjects) are given in Table 1 .

\section{TABLE1}




\section{GEOMETRICAL PARAMETERS AND RELEVANT DESIGN SPECIFICATIONS}

\begin{tabular}{|c|c|c|}
\hline S.No. & $\begin{array}{l}\text { Design geometrical } \\
\text { parameter }\end{array}$ & Design range \\
\hline 1 & Femoral head offset & $32-54 \mathrm{~mm}$ \\
\hline 2 & Femoral head diameter & $37.2-54 \mathrm{~mm}$ \\
\hline 3 & Femoral head position & $37.6-68 \mathrm{~mm}$ \\
\hline 4 & $\begin{array}{l}\text { Mediolateral Canal width, } \\
20 \mathrm{~mm} \text { above the LT }\end{array}$ & $17.3-49.5 \mathrm{~mm}$ \\
\hline 5 & $\begin{array}{l}\text { Mediolateral Canal width, } \\
\text { at the level of the LT }\end{array}$ & $14-36.6 \mathrm{~mm}$ \\
\hline 6 & $\begin{array}{l}\text { Mediolateral Canal width, } \\
20 \mathrm{~mm} \text { below the LT }\end{array}$ & $11.6-24.5 \mathrm{~mm}$ \\
\hline 7 & $\begin{array}{l}\text { Mediolateral Canal width at } \\
\text { the isthmus }\end{array}$ & $4.9-13 \mathrm{~mm}$ \\
\hline 8 & $\begin{array}{l}\text { Periosteal width at the } \\
\text { isthmus }\end{array}$ & $21.5-35 \mathrm{~mm}$ \\
\hline 9 & Isthmus position & $86-157 \mathrm{~mm}$ \\
\hline 10 & Neck-shaft angle & $100^{\circ}-135^{\circ}$ \\
\hline 11 & $\begin{array}{l}\text { Anteroposterial Canal } \\
\text { width, } 20 \mathrm{~mm} \text { above the LT }\end{array}$ & $13-38 \mathrm{~mm}$ \\
\hline 12 & $\begin{array}{l}\text { Anteroposterial Canal } \\
\text { width, at the level of the LT }\end{array}$ & $13.2-27.5 \mathrm{~mm}$ \\
\hline 13 & $\begin{array}{l}\text { Anteroposterial Canal } \\
\text { width, } 20 \mathrm{~mm} \text { above the } \mathrm{LT}\end{array}$ & $11-23 \mathrm{~mm}$ \\
\hline 15 & $\begin{array}{l}\text { Anteroposterial canal width } \\
\text { at the isthmus }\end{array}$ & $6-15.5 \mathrm{~mm}$ \\
\hline 16 & Femoral neck length & $36.3-63$ \\
\hline
\end{tabular}

TABLE 2

PHYSICAL PROPERTIES OF STEM CROSSSECTIONS

\begin{tabular}{|c|c|c|c|}
\hline $\begin{array}{c}\text { Cross- } \\
\text { section }\end{array}$ & $\begin{array}{l}\text { Stem } \\
\text { surface } \\
\left(\mathrm{mm}^{2}\right)\end{array}$ & $\begin{array}{l}\text { Stem } \\
\text { Volume } \\
\left(\mathrm{mm}^{3}\right)\end{array}$ & $\begin{array}{l}\text { Stem } \\
\text { Mass } \\
(\mathrm{gm})\end{array}$ \\
\hline Oval & 7140.89 & 34855 & 154.37 \\
\hline Wedge & 7164.51 & 29780 & 131.89 \\
\hline Trapezoidal & 8997.73 & 39932 & 176.85 \\
\hline Rectangular & 6519.35 & 21864 & 96.83 \\
\hline
\end{tabular}

Table 2 lists the dimensional properties and masses of the stems with different cross sections. The stem surface area and volume were calculated by solid model properties listed in Pro/ENGINEER. The stem surface area, volume and mass of the stem with a trapezoidal cross section were the maximum, while the corresponding values for the rectangular cross section were the minimum. The surface area of the wedge cross section $\left(7164.51 \mathrm{~mm}^{2}\right)$ was slightly more than that of the oval cross section (7140.89 $\mathrm{mm}^{2}$ ), however, the volume and mass were minimum for the wedge cross-section.

Thus the advantage of the wedge cross-section is that it provides maximum surface area, thereby allowing more bone-surface contact integration, accompanied with lower volume and mass. 


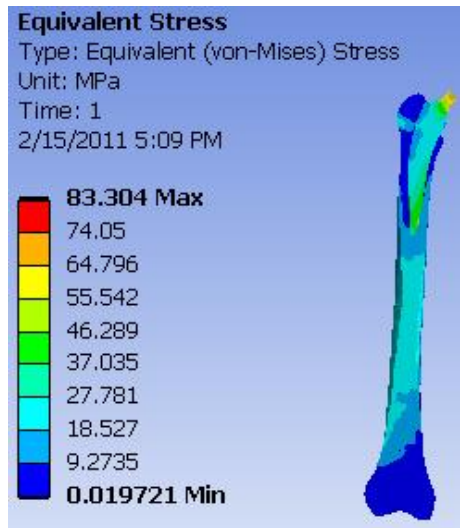

Fig. 6. Typical FEA result for a wedge cross section Fig. 7 shows a contour plot for a relative vertical sliding micromotion between the bone and implant, for the wedge cross section stem. The maximum axial micromotion of the stem in the femoral canal was found to be $0.050 \mathrm{~mm}$ $(50 \mu \mathrm{m})$.

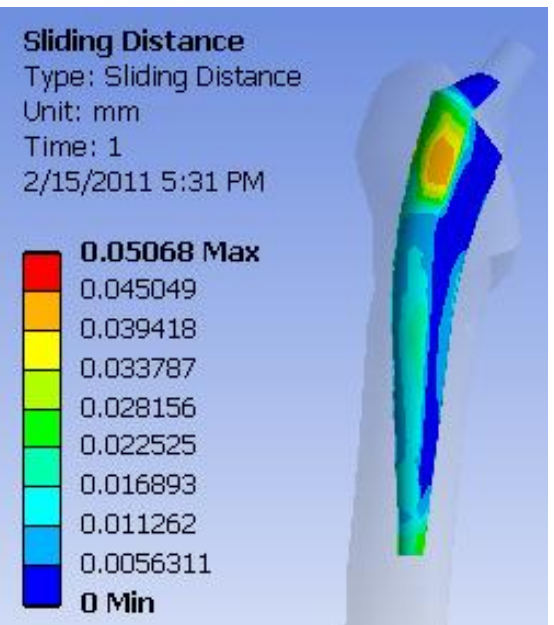

Fig. 7. Contour plot of vertical micromotions (sliding distance at the bone-implant interface) for wedge shaped implant under compressive load of $4500 \mathrm{~N}$.

Fig. 8 exhibits vertical sliding distance at the bone implant interface, as a function of applied load, for the four cross sections for the oval, wedge and rectangular cross sections. It was found that the wedge cross-section provided the greatest resistance to micromotion at the bone-implant interface under compressive loads, followed by the rectangular cross-section. The vertical micromotion is highest for the trapezoidal cross section and it is simply described as linear between 0.58 and $1.5 \mathrm{~mm}$ sliding distance the highest increase per $1000 \mathrm{~N}$ increase in load. The oval cross section also exhibited a somewhat linear increase in interface movement, with increase in load, and was higher than that of the wedge and oval cross sections. For the wedge and oval cross sections, the curves match closely. However, at certain loads $(\sim 3000 \mathrm{~N}$ and $6000 \mathrm{~N}$ in Fig. 8b), the interface movement was slightly higher for the oval cross section, which indicates the wedge to be a better option for initial primary stability as well as long term stability after osseointegration.

Fig. 9 illustrates the interface transverse sliding movement as a function of torsional load, for the four different cross sections investigated. The interfacial transverse sliding movement is the rotation of neck axis of the stem at the femoral axis due to the application of a transverse force (see Fig.1a). The transverse sliding movements are expressed as the linear shift of the center of the stem neck tip in the transverse plane keeping the femoral axis as the axis of rotation. It is seen that under torsional loads, the sliding distance is the highest for the oval cross section with the highest increase per $\mathrm{Nm}$ increase in torque. This was followed by the rectangular, wedge and trapezoidal cross sections respectively. All the curves exhibited a fairly linear relationship.

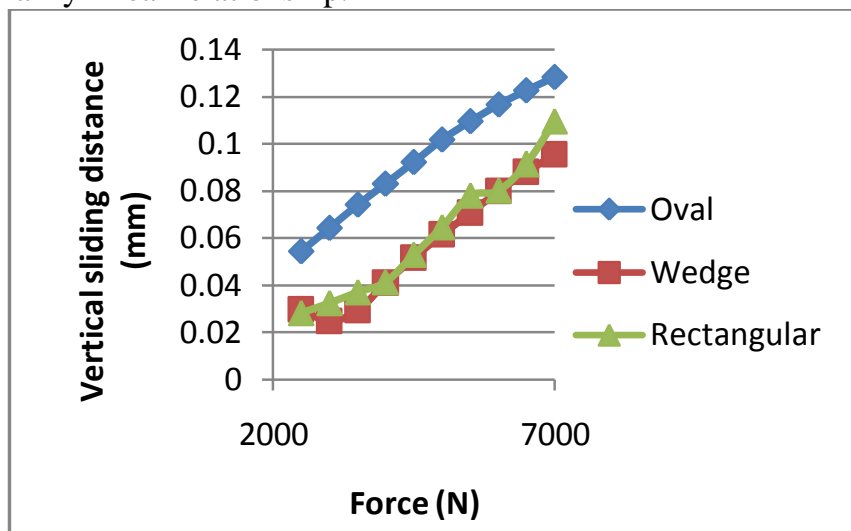

Fig. 8. Graph showing verical sliding distance under compressive force for oval, wedge and recrangular geometrical shapes



Fig. 9. Graph showing the transverse sliding distance under torque for oval, wedge, rectangular and trapezoidal geometrical shapes

\section{IV.DISCUSSION}

The geometric cross-sectional shape of a hip joint prosthesis plays a major role on the stress transfer in supporting structures. The effect of joint force due to body weight and micromotions on the interface of the prosthesis and bone were evaluated using F.E.M. Axial and torsional loading (monotonic or cyclic) has been identified as one potential mode of implant failure in THR [20]. Despite this, few studies have addressed the role that the implant stem shape may have in regards to mechanical stability under both axial and torsional loading, particularly for cementless femoral stems.

In Fig. 4, it is seen that the stresses for the wedge cross section are well within the yield point for bone (yield strength of bone is $160 \mathrm{MPa}$ [21]) as well as $\mathrm{Ti}$ alloy (yield strength of Ti6Al4V is $970 \mathrm{MPa}$ ), at all the chosen 
locations. For the other cross sections, the value is higher at location 2. Figs. 7 ( $a$ and $b$ ) indicate that, for the wedge cross section, the highest vertical motion up to a load of $7000 \mathrm{~N}$ was below the acceptable limit of $0.15 \mathrm{~mm}$ for bone ingrowth [22], while for the other cross sections, it crosses this limit. For the trapezoidal cross section, the vertical motion is the highest with a value of $1.5 \mathrm{~mm}$. However, under torsional loads, the transverse sliding distance was the minimum for the trapezoidal cross section, second lowest being for the wedge cross section. Considering the stresses and interfacial relative movements, it is proved logically that the wedge crosssection would perform the best under all physiological loading conditions.

\section{V.CONCLUSIONS}

Four geometrical cross section shapes for cementless femoral stems were analyzed for Von-Mises stresses and primary stability, under compressive and torsional loads, using a finite element approach. Loads simulating physiological activity of walking were used. Results were compared for sliding distances and stresses. The best overall performance was shown by the wedge cross sectional shape. Incorporation of a wedge cross section in standard prosthesis would therefore enhance performance, avoiding the use of more expensive modular, customized prostheses.

\section{ACKNOWLEDGMENT}

The authors wish to thank the Council of Scientific and Industrial Research (CSIR), India, NMITLI project for a generous grant to undertake this study on selected medical implants, RP01841. The authors also wish to acknowledge the support and data provided by Dr. R. Malhotra, department of orthopaedics, All India Institute of Medical Sciences (AIIMS).

\section{REFERENCES}

[1] http://arthritis.about.com/cs/jtreplace/a/cement.htm

[2] R. Huiskes, and N. Verdonschot, Biomechanics of Artificial Joints:The Hip, in: V. C. Mow and W. C. Hayes, eds., Basic Orthopaedic Biomechanics,2nd ed., Lippincott-Raven, Philadelphia, pp. 395-460, 1997.

[3] E.I. Najjar, and E.R. McWilliams, Forensic Anthropology: The structure, Morphology and variations of human bone and dentition, Springfield, IL: Charles C Thomas, 1978.

[4] M.F. Ericksen, Ageing changes in the medullary cavity of the proximal femur in American black and whites, Am. J. Phys. Anthropol., 51, pp. 563-569, 1979

[5] R.C. Siwach, and S. Dahiya, Anthropometric study of proximal femur geometry and its clinical application, Ind. J. Orthop., 37(4), pp. 247-251, 2003

[6] V.S. Reddy, G.V.S., Moorthy, and S.G. Reddy, Do we need a special design of femoral component of total hip prosthesis in our patients? Ind. J. Orthop., 33(4), pp. 282-4, 1999

[7] K. Leung, P. Procter, B. Robioneck, and K. Behrens, Geometric mismatch of the gamma nail to the Chinese femur, Clin. Orthop., 323, pp. 42-48, 1996.

[8] Pyburn, E, Goswami, T., 2004, "Finite element analysis of femoral components paper III - hip joints," Mater Des, 25, pp. 705-13.

[9] B. Latham, and T. Goswami, Effect of geometric parameters in the design of hip implants paper IV. Mater Des., 25, pp. 715-22, 2004.

[10] A.L. Sabatini, and T. Goswami, Hip implants VII: Finite element analysis and optimization of cross-sections. Mater Des., 29, pp. 1438-1446, 2008
[11] R. Huiskes, R. Boeklagen, Mathematical shape optimization of hip prosthesis design, J. Biomech., 22, pp. 793 -804, 1989.

[12] R.J. Yang, K.K. Choi, R.D. Crowninshield, and R.A. Brand, Design sensitivity analysis: a new method for implant design and a comparison with parametric finite element analysis, J. Biomech., 17, pp. $849-854,1984$.

[13] S. Y. Yoon, G.H. Jang, and Y.Y. Kim, Shape optimal design of the stem of a cemented hip prosthesis to minimize stress concentration in the cement layer, J. Biomech., 22, pp. 1279 -1284, 1989.

[14] P. Kowalczyk, Design optimization of cementless femoral hip prostheses using finite element analysis, J. Biomech. Eng., 123, pp. 396-402, 2001.

[15] R.B. Ruben, J. Folgado, and P.R. Fernandes, Shape optimization of uncemented hip prostheses, Virtual and Physical Prototyping, 1(3), pp. 147-158, 2006.

[16] F. Adam, D.S. Hammer, D. Pape, and D. Kohn, Femoral anatomy, computed tomography and computer-aided design of prosthetic implants, Arch Orthop Trauma Surg, 122, pp. 262-268, 2002.

[17] C.A. Engh, D. O'Connor, M. Jasty, T.F. McGovern, J.D. Bobyn, and W.H. Harris, Quantification of implant micromotion, strain shielding, and bone resorption with porous-coated anatomic medullary locking femoral prostheses, Clinical Orthopaedics, 285, pp. 13-29, 1992.

[18] H.F. El-Sheikh, B. MacDonalds, M.S.J. Hashmi, Material selection in the design of the femoral component of cemented total hip replacement, J. Mater Process Technol, 122, pp. 309-17, 2002.

[19] D. Bennett, and T. Goswami, Finite element analysis of hip stem designs, Materials and Design 29, pp. 45-60, 2008.

[20] G. Bergmann, F. Graichen, and A. Rohlmann, Is Staircase Walking a Risk for the Fixation of Hip Implants? J. Biomech., 28(5), pp. 535-553, 1995.

[21] H. H. Bayraktar, E.F. Morgan, G.L. Niebur, et al., Comparison of the elastic and yield properties of human femoral trabecular and cortical bone tissue, Journal of Biomechanics, 37, pp. 27-35, 2004

[22] K. Søballe, E.S. Hansen, H. Brockstedt-Rasmussen, C.M. Pedersen, and C. Bünger, Hydroxyapatite coating enhances fixation of porous coated implants: a comparison in dogs between press fit and noninterference fit, Acta Orthop Scand 61, pp. 299-306, 1990. 